\title{
Simulation of Superconducting Single Photon Detector coupled with Metal-Insulator-Metal Circular Grating
}

\author{
Shao-Wei Wang ${ }^{1,2 *}$, Feiliang Chen ${ }^{1}$, Ying Zhang ${ }^{3}$, Qian $\mathrm{Li}^{1}$, Xiaolan $\mathrm{Sun}^{3}$, Xiaoshuang Chen ${ }^{1}$, Wei $\mathrm{Lu}^{1,2 *}$ \\ ${ }^{1}$ National Lab for Infrared Physics, Shanghai Institute of Technical Physics, Chinese Academy of Sciences, 500 Yu Tian Road, \\ Shanghai 200083, China \\ ${ }^{2}$ Shanghai Advanced Research Institute, Chinese Academy of Sciences, 99 Hai-Ke Road, Shanghai 201210, China \\ ${ }^{3}$ Key Laboratory of Specialty Fiber Optics and Optical Access Networks, Shanghai University, Shanghai 200072, China
}

\begin{abstract}
Metal-insulator-metal (MIM) circular grating structure has been introduced to couple with superconducting nanowire single-photon detector (SNSPD) to enhance its response. According to our numerical simulation results, the coupling efficiency at $1.55 \mu \mathrm{m}$ can be enhanced by MIM circular grating and improving the response of SNSPD obviously. About 19.5 times of enhancement in response can be obtained with proper structure parameters.
\end{abstract}

\section{INTRODUCTION}

Single photon detection is a key technology for weak light detection and photon counting. It plays an important role in optical communication, quantum information processing, astronomy, and many other fields [1]. Niobium nitride $(\mathrm{NbN})$ superconducting nanowire single-photon detectors (SNSPDs) [2] are an ultrasensitive photon counting technology at infrared wavelengths, which has good detection efficiency, low darkcount rate, and high speed. However, the thickness of superconducting $\mathrm{NbN}$ layer is very thin, even less than $10 \mathrm{~nm}$. Therefore, the detection efficiency of SNSPDs is not only limited primarily by optical loss due to the reflection from, also transmission through the device.

To solve the problem of optical loss, approaches are investigated to enhance the coupling efficiency of light and increase the response of devices. Kristine M. Rosfjord et al. demonstrated maximum detection efficiency of $57 \%$ at 1550 $\mathrm{nm}$ wavelength by adding an optical cavity and anti-reflection coating to a nanowire photodetector [3].

In this work, we propose a metal-insulator-metal (MIM) [4,5] circular grating structure to couple with SNSPD and enhance its response by collecting photons from large area to the limited active area.

\section{SiMULATION MODELS}

All the simulations are conducted by Finite Difference Time Domain (FDTD). The structure we studied is composed of SNSPD structure and MIM circular grating structure as shown in figure 1. The MIM circular grating structure can collect the photons in a very large area compared to the active area of SNSPD itself, and focus onto the detection area. Equivalently, the photon density detected by SNSPD increases and the response enhanced remarkably.

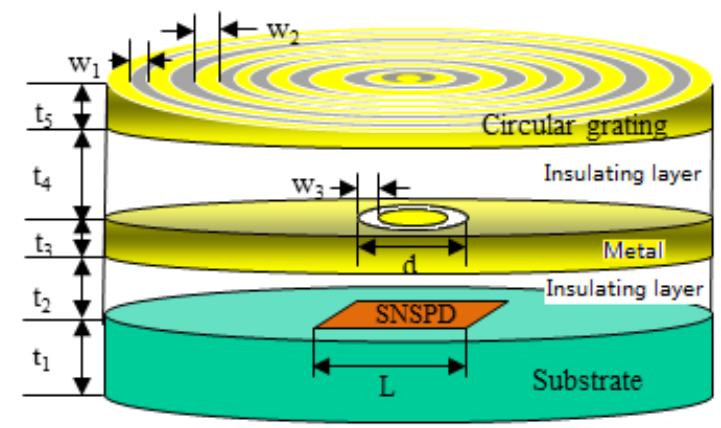

(a)

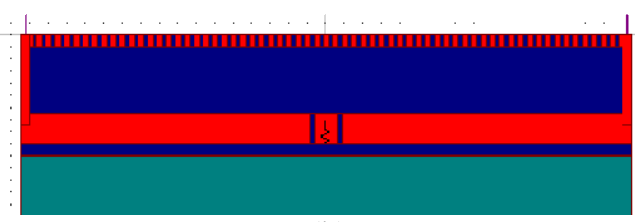

(b)

Figure 1 (a) 3D schematic diagrams and (b) Cross-sectional view of SNSPD integrated with MIM circular grating.

For example in more detail, the substrate can be $\mathrm{MgO}$, sapphire, or even Si with thickness of $t_{1}$. The SNSPD is composed of $\mathrm{NbN}$ superconductor nanowire wound in a tight meander pattern to maximize the effective area. The active area is chosen as $10 \mu \mathrm{m} \times 10 \mu \mathrm{m}$ in our simulation. The bottom of MIM structure is a transparent ring cavity in a $t_{3}$ thick metal film with external and inner diameters of $d$ and $d-2 w_{3}$, respectively. The thickness of insulating layer in the MIM structure is $t_{4}$. The top of MIM structure is a sub-wavelength circular grating

\footnotetext{
*Corresponding authors: wangshw@mail.sitp.ac.cn, luwei@mail.sitp.ac.cn
} 
consisting of a series of concentric rings, those widths of rings and gaps in between are $w_{2}$ and $w_{1}$ with thickness of $t_{5}$. An insulating layer with thickness of $t_{2}$ is inserted to avoid the conducting between the SNSPD and MIM structure. In the simulation, metal and insulating layers mentioned above are chosen as $\mathrm{Ag}$ and $\mathrm{SiO}_{2}$, respectively. The parameters of $t_{1}, t_{2}, t_{3}$, $t_{4}, t_{5}, d, w_{3}$ are set as $400,0.1,0.3,1.15,0.2,3.5$, and $1.0 \mu \mathrm{m}$, respectively. The width and gap of concentric rings $w_{1}$ and $w_{2}$ are investigated, according with the periodicity, to obtain a high coupling efficiency.

\section{RESUlTS AND Discussion}

Figure 2 shows the transmission spectrum of MIM circular grating. There is a transmission peak centered at $1.55 \mu \mathrm{m}$ with suppressing background light of other wavelengths.

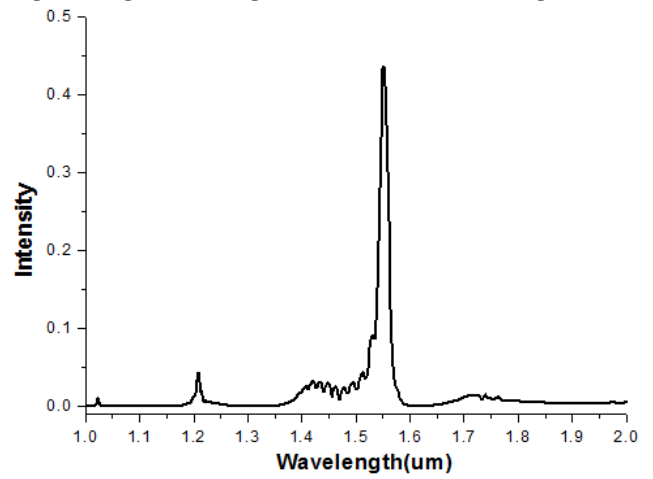

Figure 2 Transmission spectrum of MIM circular grating $\left(\mathrm{w}_{1}=0.6 \mu \mathrm{m}\right.$, $\mathrm{w}_{2}=0.5 \mu \mathrm{m}$, number of periods is 82 )

The response enhance factor was calculated by comparing the optical power density reaching SNSPDs integrated with and without MIM circular grating. Figure 3(a) shows that the calculated enhancing factor as a function of the width of grating gap $\mathrm{w}_{1}\left(\mathrm{w}_{2}=0.5 \mu \mathrm{m}\right.$, number of periods is 82$)$. A maximum enhancing factor of 12.1 can be obtained with gap width of $0.6 \mu \mathrm{m}$. Figure 3(b) shows that the change of enhancing factor with the gap width $\mathrm{w}_{2}\left(\mathrm{w}_{1}=0.6 \mu \mathrm{m}\right.$, number of periods is 82 ) changing from 0.3 to $0.7 \mu \mathrm{m}$. The maximum enhancing factor is 12.1 as well when the gap width $w_{2}$ is $0.5 \mu \mathrm{m}$.
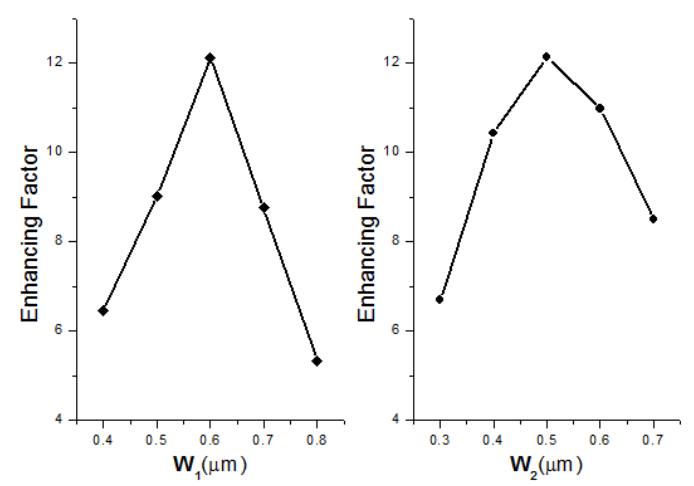

Figure 4 (Left) Enhancing factor VS gap width of grating $\mathrm{w}_{1}\left(\mathrm{w}_{2}=0.5\right.$ $\mu \mathrm{m}$, number of periods is 82). (Right) Enhancing factor VS Ag width of grating $\mathrm{w}_{2}\left(\mathrm{w}_{1}=0.6 \mu \mathrm{m}\right.$, number of periods is 82$)$
The influence of circular grating periodicity on the enhancing factor is studied as well. The change of enhancing factor with the number of periods from 40 to 240 is shown in Fig. 4, keeping $\mathrm{w}_{1}$ and $\mathrm{w}_{2}$ constant. The enhancing factor increases remarkably when the periodicity is less than 160 . It tends to be constant and saturated when more than 160. 19.5 times of enhancement can be obtained when the number of periods is bigger than 200 .

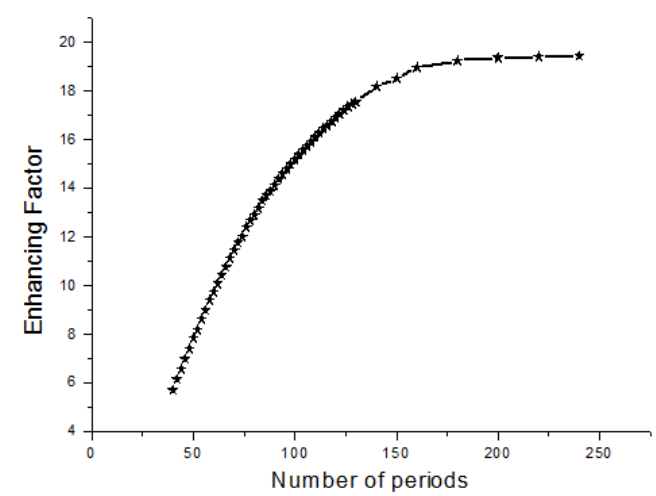

Figure 4 Change of enhancing factor with the number of grating periodicity $\left(\mathrm{w}_{1}=0.6 \mu \mathrm{m}, \mathrm{w}_{2}=0.5 \mu \mathrm{m}\right)$

\section{SUMMARY AND CONCLUSIONS}

SNSPD coupled with MIM circular grating structure has been investigated numerically. The MIM circular grating structure can collect the photons in a very large area and couple to the SNSPD. That is to say, the photon density detected by SNSPD increases and enhances the response. The simulation results show that the response of SNSPD at $1.55 \mu \mathrm{m}$ can be enhanced remarkably in aid of MIM circular grating structure. The enhancement of response can be as high as 19.5 times when choosing proper structure parameters.

\section{ACKNOWLEDGEMENTS}

This work was supported by the Shanghai Science and Technology Foundations (11nm0502100, 11dz2283100, 12nm0502900, 12dz2293600).

\section{REFERENCES}

[1] Wenjuan Wang, Shao-Wei Wang, Wei Lu, Feiliang Chen, Ying Zhang, Xiaolan Sun, Ning Li, Zhifeng Li, and Xue Li, Laser selective focusing utilized for remarkablely inhancing the response of photodetectors, Infrared and Laser Engineering 9, xxx (2013).

[2] Kristine M. Rosfjord1, Joel K. W. Yang, Eric A. Dauler, Andrew J. Kerman, Vikas Anant, Boris M. Voronov, Gregory N. Gol'tsman,Karl K. Berggren, Nanowire Single-photon detector with an integrated optical cavity and anti-reflection coating, Optics Express 14, 527 (2006).

[3] Zhen Wang, Shigehito Miki, and Mikio Fujiwara, Efficiently Coupling Light to Superconducting Nanowire Single-Photon Detectors, IEEE J. of selected topics in quantum electronics 15, 336 (2009)

[4] Zhanghua Han, Erik Forsberg \&Sailing He, Surface Plasmon Bragg Gratings Formed in Metal-Insulator-Metal Waveguides, IEEE Photoncs Technology Letters 19, 91 (2007).

[5] Barnes W L, Dereux A, Ebbesen T W, Surface plasmon subwavelength optics, Natrue 424, 824 (2003). 